\title{
Reflection of micropolar elastic waves at the non-free surface of a micropolar elastic half-space
}

Received: 4 September 2014 / Revised: 28 January 2015 / Published online: 1 May 2015

(C) Springer-Verlag Wien 2015

\begin{abstract}
The reflection problem of the micropolar elastic waves at the non-free surface of a micropolar elastic half-space is studied in this paper. Different from the classic elastic solid, there are four kinds of elastic waves in the micropolar elastic solid and three of them are dispersive. The boundary conditions at the non-free surface of a micropolar elastic half-space are used to obtain the linear algebraic equation sets from which the amplitude ratios of reflection waves to the incident wave can be determined. Then, the reflection coefficients in terms of energy flux ratios are calculated numerically, and the normal energy flux conservation is used to validate the numerical results. At last, the influence of the boundary parameters, which reflect the mechanical behavior of the non-free surface, on the reflection energy partition of micropolar elastic waves is discussed based on the numerical results. Two cases of incident longitudinal displacement wave and incident coupled transverse displacement and transverse microrotational wave are considered.
\end{abstract}

\section{Introduction}

A micropolar elastic solid differs from a classical elastic solid in that each point has an extra microrotational degree of freedom besides the translational degree of freedom and therefore applies to model the mechanical behavior of the structure material with fibrous or granular microstructure. Eringen [3] proposed and developed the theory of micropolar elastic solids. Smith [8], Parfitt and Eringen [6] and Ariman [1] studied early the wave propagation in the micropolar elastic solids. It was found that there are four types of elastic waves: the longitudinal displacement wave (LD wave); longitudinal microrotational wave (LR wave); and two sets of coupled transverse displacement wave and transverse microrotational wave (CTDR wave). The transverse displacement wave is similar to the classical shear wave and will reduce to it in the limit of classical elasticity. The appearance of a transverse microrotational wave coupled with the transverse displacement wave is new. More interesting is that the amplitude vectors of the transverse displacement wave and the transverse microrotational wave, and the wave vector are mutually perpendicular. Because the micropolar solid can bear couple stress in addition to the force stress, and microrotation in addition to the translational displacement, the boundary conditions of a micropolar elastic solid and the interface condition at two different micropolar solids are different from the classic elastic materials. Using the appropriate boundary conditions, [6] studied the reflection problem of micropolar waves from a free surface of a micropolar half-space and [1] studied the reflection problem from a fixed surface of a micropolar half-space and discussed some special cases of reflection waves. Tomar and Gogna [9-11] further studied the reflection and transmission problem of

P. Zhang · P. Wei $(\varangle)$

Department of Applied Mechanics, University of Sciences and Technology Beijing, Beijing 100083, China

E-mail:weipj@ustb.edu.cn

P. Wei $\cdot$ Q. Tang

State Key Laboratory of Nonlinear Mechanics (LNM), Chinese Academy of Science, Beijing 100080, China 
micropolar elastic waves at the interface between two different micropolar solids. Similar problem for the interface between liquid and micropolar solid was studied by [12]. Recently, Singh [7] also studied the wave propagation in the anisotropic micropolar elastic solid, and Khurana and Tomar [4,13] studied the electromechanical coupling effects on the wave propagation in an electric-micropolar elastic solid. Moreover, [2] also studied the Rayleigh waves in a micropolar elastic solid. Because the reflection and transmission problem is of special importance in nondestructive tests (NDT), it therefore attracts special attentions from many researchers. In the above-mentioned works, the interface between two different micropolar solids is usually assumed to be perfect, namely the weld contact interface, and the boundary surface where the micropolar elastic waves reflect is usually assumed to be free surface, that is, traction-free surface. However, the interface may be imperfect due to interface accumulative damage [5], and the boundary surface may be non-free but with various constraints in actual engineering problem.

In this paper, the reflection problem of micropolar elastic waves at the non-free surface of a micropolar half-space is studied. The non-free surface is modeled as a surface with distributed elastic constraint or support. Each mass point at the surface is subjected to the normal and tangent translational constraint and the rotational constraint. Three boundary parameters are introduced to represent respective constraint degree. The reflection coefficients in terms of energy flux ratios are calculated from the boundary condition of non-free surface, and the influences of boundary parameters on the reflection energy flux partitions of various waves are discussed based on the numerical results. The results of corresponding problem for free surface as well as the fixed surface can be recovered from the present surface model.

\section{Statements of problem}

The motion equations of mass point in the micropolar elastic solid can be expressed as (see [6])

$$
\begin{aligned}
\left(c_{1}^{2}+c_{3}^{2}\right) \nabla(\nabla \cdot \mathbf{u})-\left(c_{1}^{2}+c_{3}^{2}\right) \nabla \times(\nabla \times \mathbf{u})+c_{3}^{2} \nabla \times \boldsymbol{\varphi} & =\ddot{\mathbf{u}}, \\
\left(c_{4}^{2}+c_{5}^{2}\right) \nabla(\nabla \cdot \varphi)-c_{4}^{2} \nabla \times(\nabla \times \boldsymbol{\varphi})+\omega_{0}^{2} \nabla \times \mathbf{u}-2 \omega_{0}^{2} \boldsymbol{\varphi} & =\ddot{\boldsymbol{\varphi}},
\end{aligned}
$$

where $c_{1}^{2}=(\lambda+2 \mu) / \rho, c_{2}^{2}=\mu / \rho, c_{3}^{2}=K / \rho, c_{4}^{2}=\gamma / \rho j, c_{5}^{2}=(\alpha+\beta) / \rho j, \omega_{0}^{2}=c_{3}^{2} / j=K / \rho j . \lambda$ and $\mu$ are the Lamé constants. $K, \alpha, \beta$, and $\gamma$ are the micropolar elastic moduli. $\rho$ is the mass density, and $j$ is the microinertia. $\mathbf{u}$ and $\varphi$ are the displacement vector and the microrotational vector, respectively. Here, the body force and the body couples are assumed to be zero. In order to uncouple Eq. (1), we represent $\mathbf{u}$ and $\varphi$ with a scalar potential and a vector potential by the application of Helmholtz decomposition of a vector, namely

$$
\begin{aligned}
& \mathbf{u}=\nabla q+\nabla \times \mathbf{U}, \nabla \cdot \mathbf{U}=0 \\
& \boldsymbol{\varphi}=\nabla \xi+\nabla \times \boldsymbol{\Phi}, \nabla \cdot \boldsymbol{\Phi}=0
\end{aligned}
$$

Inserting Eq. (2) into Eq. (1) leads to

$$
\begin{gathered}
\left(c_{1}^{2}+c_{3}^{2}\right) \nabla^{2} q=\ddot{q}, \\
\left(c_{4}^{2}+c_{5}^{2}\right) \nabla^{2} \xi-2 \omega_{0}^{2} \xi=\ddot{\xi}, \\
\left(c_{2}^{2}+c_{3}^{2}\right) \nabla^{2} \mathbf{U}+c_{3}^{2} \nabla \times \boldsymbol{\Phi}=\ddot{\mathbf{U}}, \\
c_{4}^{2} \nabla^{2} \boldsymbol{\Phi}+\omega_{0}^{2} \nabla \times \mathbf{U}-2 \omega_{0}^{2} \boldsymbol{\Phi}=\ddot{\boldsymbol{\Phi}} .
\end{gathered}
$$

The solution of Eq. (3) can be expressed as

$$
(q, \xi, \mathbf{U}, \boldsymbol{\Phi})=(a, b, \mathbf{A}, \mathbf{B}) \exp [i k(\mathbf{n} \cdot \mathbf{r}-V t)],
$$

where $a, b, \mathbf{A}$, and $\mathbf{B}$ are the complex amplitudes of four waves, respectively. $V$ is the wave speed, $k$ is the wave number, $\mathbf{n}$ is the unit vector of propagation direction, $\mathbf{r}$ is the position vector. Inserting Eq. (4) into Eq. (3), the dispersive relations of four basic waves are obtained (see [6]).

(a) The longitudinal displacement wave (LD wave) reflects the nature of the physical field $q$. The wave speed is $V_{1}=\sqrt{(\lambda+2 \mu+K) / \rho}$. It is a non-dispersive wave.

(b) The longitudinal microrotational wave (LR wave) reflects the nature of the physical field $\xi$. The wave speed is $V_{2}=\sqrt{(\alpha+\beta+\gamma) / \rho j\left(1-2 K / \rho j \omega^{2}\right)}$. 
(c) Two sets of coupled transverse displacement wave and transverse microrotational wave (CTDR3 wave with speed $\mathrm{V}_{3}$ and $\mathrm{CTDR} 4$ wave with speed $\mathrm{V}_{4}$ ) consist of the transverse displacement field $\mathbf{U}$ coupled with the transverse microrotational field $\boldsymbol{\Phi}$. Their wave speeds are

$$
V_{3,4}^{2}=\frac{1}{2(1-\eta)}\left[c_{4}^{2}+c_{3}^{2}+c_{2}^{2}-\left(c_{2}^{2}+c_{3}^{2} / 2\right) \eta \pm \Delta\right]
$$

where

$$
\Delta=\left\{\left[\left(c_{4}^{2}-c_{2}^{2}-c_{3}^{2}\right)+\left(c_{2}^{2}+c_{3}^{2} / 2\right) \eta\right]^{2}+2 c_{3}^{2} c_{4}^{2} \eta\right\}^{1 / 2}, \quad \eta=2 \omega_{0}^{2} / \omega^{2}
$$

It is noted that $V_{2}$ and $V_{3}$ are nonnegative only when $\omega>\sqrt{2} \omega_{0}$. Therefore, $\omega_{c}=\sqrt{2} \omega_{0}$ is the cutoff frequency of the LR wave and the CTDR3 wave. It should be pointed out that the amplitude vectors A and $\mathbf{B}$ are orthogonal to each other and both are orthogonal to the propagation direction $\mathbf{n}$ of the coupled waves. The explicit relation between them is

$$
\mathbf{B}=-\frac{i \omega_{0}^{2}}{k_{p}\left(V_{p}^{2}-2 \omega_{0}^{2} / k_{p}^{2}-c_{4}^{2}\right)} \mathbf{n} \times \mathbf{A},
$$

where $p=3,4$ stands for CTDR3 and CTDR4 wave, respectively.

Moreover, an important difference between CTDR3 wave and CTDR4 wave, which was neglected by previous references, is that the transverse microrotational field dominates in CTDR3 wave while the transverse displacement field dominates in CTDR4 wave. In order to show this difference clearly, the amplitude ratio $|\mathbf{B} / \mathbf{A}|$ of two sets of coupled waves for the micropolar material whose material parameter is given in the next section is shown in Fig. 2. It is noted that this difference becomes more evident as the circular frequency increases.

Let the oxy coordinate plane be the non-free surface of a micropolar elastic half-space, see Fig. 1. An incident wave travels along the direction $\mathbf{n}_{\mathbf{0}}$ in the half-space with incident angle $\theta_{0}$ and impinges the non-free surface $z=0$. We consider only the plane strain case, namely

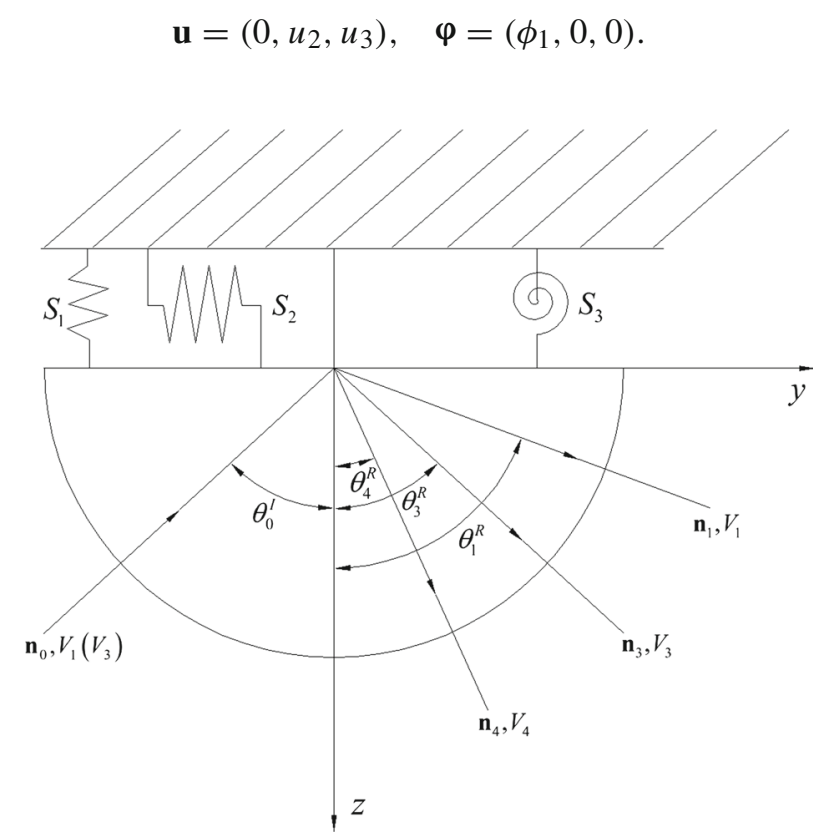

Fig. 1 Reflection of micropolar elastic waves from a non-free surface of a micropolar elastic half-space 


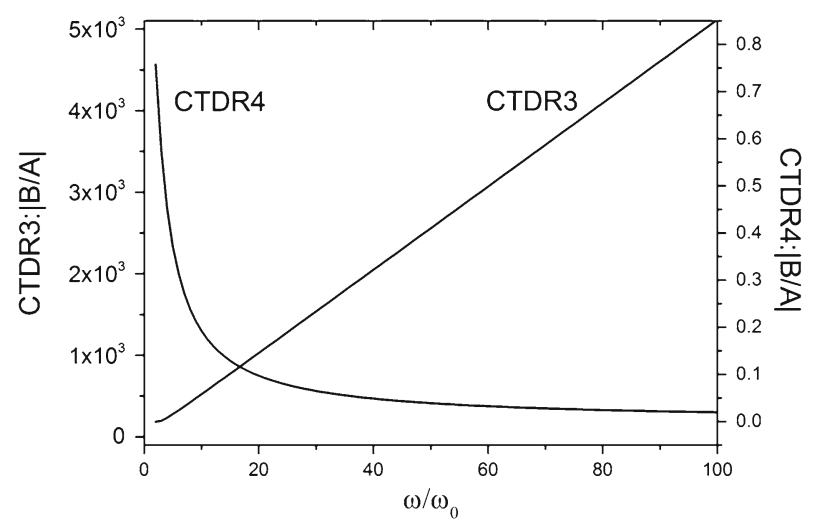

Fig. 2 Amplitude ratios of the transverse microrotational field to the transverse displacement field in two sets of coupled waves

It is noticed that the longitudinal rotational wave (LR) cannot appear in the case. The incident wave (indicated by superscript " $I$ ") and the reflection waves (indicated by superscript " $R$ ") can be expressed as

$$
\begin{aligned}
q^{I} & =a_{1}^{I} \exp \left[i k_{1}^{I}\left(\sin \theta_{1}^{I} y-\cos \theta_{1}^{I} z\right)-i \omega_{1}^{I} t\right], \\
\mathbf{U}_{p}^{I} & =A_{p x}^{I} \mathbf{i} \exp \left[i k_{p}^{I}\left(\sin \theta_{p}^{I} y-\cos \theta_{p}^{I} z\right)-i \omega_{p}^{I} t\right], \\
\boldsymbol{\Phi}_{p}^{I} & =\left[B_{p y}^{I} \mathbf{j}+B_{p z}^{I} \mathbf{k}\right] \exp \left[i k_{p}^{I}\left(\sin \theta_{p}^{I} y-\cos \theta_{p}^{I} z\right)-i \omega_{p}^{I} t\right], \\
q^{R} & =a_{1}^{R} \exp \left[i k_{1}^{R}\left(\sin \theta_{1}^{R} y+\cos \theta_{1}^{R} z\right)-i \omega_{1}^{R} t\right], \\
\mathbf{U}_{p}^{R} & =\mathbf{i} A_{p x}^{R} \exp \left[i k_{p}^{R}\left(\sin \theta_{p}^{R} y+\cos \theta_{p}^{R} z\right)-i \omega_{p}^{R} t\right], \\
\boldsymbol{\Phi}_{p}^{R} & =\left[B_{p y}^{R} \mathbf{j}+B_{p z}^{R} \mathbf{k}\right] \exp \left[i k_{p}^{R}\left(\sin \theta_{p}^{R} y+\cos \theta_{p}^{R} z\right)-i \omega_{p}^{R} t\right],
\end{aligned}
$$

where $\omega=k V, p=3$ stands for the CTDR3 wave and $p=4$ stands for the CTDR4 wave. $\mathbf{i}, \mathbf{j}$, and $\mathbf{k}$ are the unit vectors of the coordinate axes.

The constitutive relation of micropolar solid can be expressed as

$$
\begin{aligned}
t_{k l} & =\lambda u_{r, r} \delta_{k l}+\mu\left(u_{k, l}+u_{l, k}\right)+K\left(u_{l, k}-\varepsilon_{k l r} \phi_{r}\right), \\
m_{k l} & =\alpha \phi_{r, r} \delta_{k l}+\beta \phi_{k, l}+\gamma \phi_{l, k},
\end{aligned}
$$

where $t_{k l}$ and $m_{k l}$ are the force stress components and the couple stress components, respectively. Inserting Eqs. (6) and (7) into Eq. (8), we obtain

$$
\begin{aligned}
t_{z z} & =\lambda \nabla^{2} q+(2 \mu+K)\left(q_{, z z}-U_{x, y z}\right), \\
t_{z y} & =\mu\left(q_{, z y}-U_{x, y y}\right)+(\mu+K)\left(q, y z+U_{x, z z}\right)+K\left(\Phi_{z, y}-\Phi_{y, z}\right), \\
m_{z x} & =\gamma\left(\Phi_{z, y z}-\Phi_{y, z z}\right) .
\end{aligned}
$$

These force stress and couple stress components are zero for the free surface. But they may have finite value and are proportionate to the displacement components and the rotational components for the non-free surface, namely

$$
\left[\begin{array}{c}
t_{z z} \\
t_{z y} \\
m_{z x}
\end{array}\right]=-i\left[\begin{array}{ccc}
S_{1} & 0 & 0 \\
0 & S_{2} & 0 \\
0 & 0 & S_{3}
\end{array}\right]\left[\begin{array}{l}
u_{z} \\
u_{y} \\
\phi_{x}
\end{array}\right],
$$

where $S_{1}, S_{2}$, and $S_{3}$ are the proportional coefficients which reflect the stiffness of the normal, tangent, and rotational elastic support. When $S_{1}, S_{2}$, and $S_{3}$ all trend to zero, the free surface is recovered. When $S_{1}, S_{2}$, and $S_{3}$ all trend to infinite, the fixed surface is recovered. The free surface and the fixed surface are two extreme cases of the non-free surface. In general, $S_{1}, S_{2}$, and $S_{3}$ take certain finite values and model the constraint 
surface. Recall that the displacement field and stress field are expressed in terms of complex quantities, it is noted there is a phase shift between the stress field and the displacement field due to the mathematical operation $u_{i, j}=i k_{j} u_{i}$. The extra phase shift does not exist, in fact. Therefore, a negative imaginary unit, $-i$, is introduced into the right side of Eq. (10) to rectify the phase shift.

In order to satisfy the boundary conditions at any boundary point and at any time, the following relations are necessary:

$$
\begin{aligned}
\omega_{p}^{I} & =\omega_{p}^{R} \quad(p=1,3,4), \\
k_{p}^{I} \sin \theta_{p}^{I} & =k_{p}^{R} \sin \theta_{p}^{R} \quad(p=1,3,4) .
\end{aligned}
$$

Equation (11) means that the reflection waves have the same circle frequency with the incident waves. Equation (12) means that the apparent wave number of every wave is same. This is same as in the classic elastic solid. After inserting Eq. (9) into Eq. (10), the boundary conditions at the non-free surface can be expressed in the form of matrix

$$
\mathbf{D} \cdot \mathbf{Z}=\mathbf{E},
$$

where $\mathbf{Z}=\left(\mathrm{z}_{1}, \mathrm{z}_{2}, \mathrm{z}_{3}\right)^{T}$ is a column matrix which consist of the amplitude ratio of reflection waves with respect to the incident wave, namely

$$
z_{1}=\frac{a_{1}^{R}}{a_{1}^{I}}, \quad z_{2}=\frac{A_{3 x}^{R}}{a_{1}^{I}}, \quad z_{3}=\frac{A_{4 x}^{R}}{a_{1}^{I}}
$$

for incident longitudinal displacement wave (LD wave), and

$$
z_{1}=\frac{a_{1}^{R}}{A_{p x}^{I}}, \quad z_{2}=\frac{A_{3 x}^{R}}{A_{p x}^{I}}, \quad z_{3}=\frac{A_{4 x}^{R}}{A_{p x}^{I}}
$$

for incident coupled transverse displacement and transverse rotational wave ( $p=3$ or 4 ). $\mathbf{E}$ is the column matrix, which is only related with the incident wave:

$$
\mathbf{E}=\left(\begin{array}{l}
{\left[\lambda+(2 \mu+K) \cos ^{2} \theta_{1}^{I}\right]\left(k_{1}^{I}\right)^{2}-\cos \theta_{1}^{I} k_{1}^{I} S_{1}} \\
(2 \mu+K) \sin \theta_{1}^{I} \cos \theta_{1}^{I}\left(k_{1}^{I}\right)^{2}-\sin \theta_{1}^{I} k_{1}^{I} S_{2} \\
0
\end{array}\right)
$$

for incident longitudinal displacement wave (LD wave), and

$$
\mathbf{E}=\left(\begin{array}{l}
(2 \mu+K) \sin \theta_{p}^{I} \cos \theta_{p}^{I}\left(k_{p}^{I}\right)^{2}-\sin \theta_{p}^{I} k_{p}^{I} S_{1} \\
-\left[\left(\mu \cos 2 \theta_{p}^{I}+K \cos ^{2} \theta_{p}^{I}\right)\left(k_{p}^{I}\right)^{2}+\frac{K \omega_{0}^{2}}{V_{p}^{2}-2 \omega_{0}^{2} /\left(k_{p}^{I}\right)^{2}-c_{4}^{2}}\right]+\cos \theta_{p}^{I} k_{p}^{I} S_{2} \\
-\frac{\gamma \omega_{0}^{2} \cos \theta_{p}^{I} k_{p}^{I}}{V_{\rho}^{2}-2 \omega_{0}^{2} /\left(k_{p}^{I}\right)^{2}-c_{4}^{2}}+\frac{\omega_{0}^{2} S_{3}}{V_{p}^{2}-2 \omega_{0}^{2} /\left(k_{p}^{I}\right)^{2}-c_{4}^{2}}
\end{array}\right)
$$

for incident coupled transverse displacement wave and transverse microrotational wave $(p=3,4)$. The explicit expressions of each element in matrix $\mathbf{D}$ are listed in Appendix A. As two extreme cases, the free surface and the fixed surface get special concerns. The explicit expressions of matrix $\mathbf{D}$ and $\mathbf{E}$ for the two extreme cases are also provided in Appendix A. Due to relative simplification, the amplitude ratios of reflection wave to incident wave can be further deduced. It is noted that the amplitude ratios for free surface reduce to Eqs. (78) and (79) in [6] and the amplitude ratio for fixed surface reduces to Eq. (45) in [1]. From Eq. (13), the amplitude ratios of reflection waves to incident waves for a non-free surface can be obtained for any given incident angle and frequency.

In order to investigate the energy partition between various reflection waves, the energy fluxes carried by the incident wave and the various reflected waves are estimated by

$$
P_{i}(t)=\frac{1}{2}\left(\dot{u}_{j} \sigma_{j i}+\dot{\varphi}_{j} m_{i j}\right)
$$



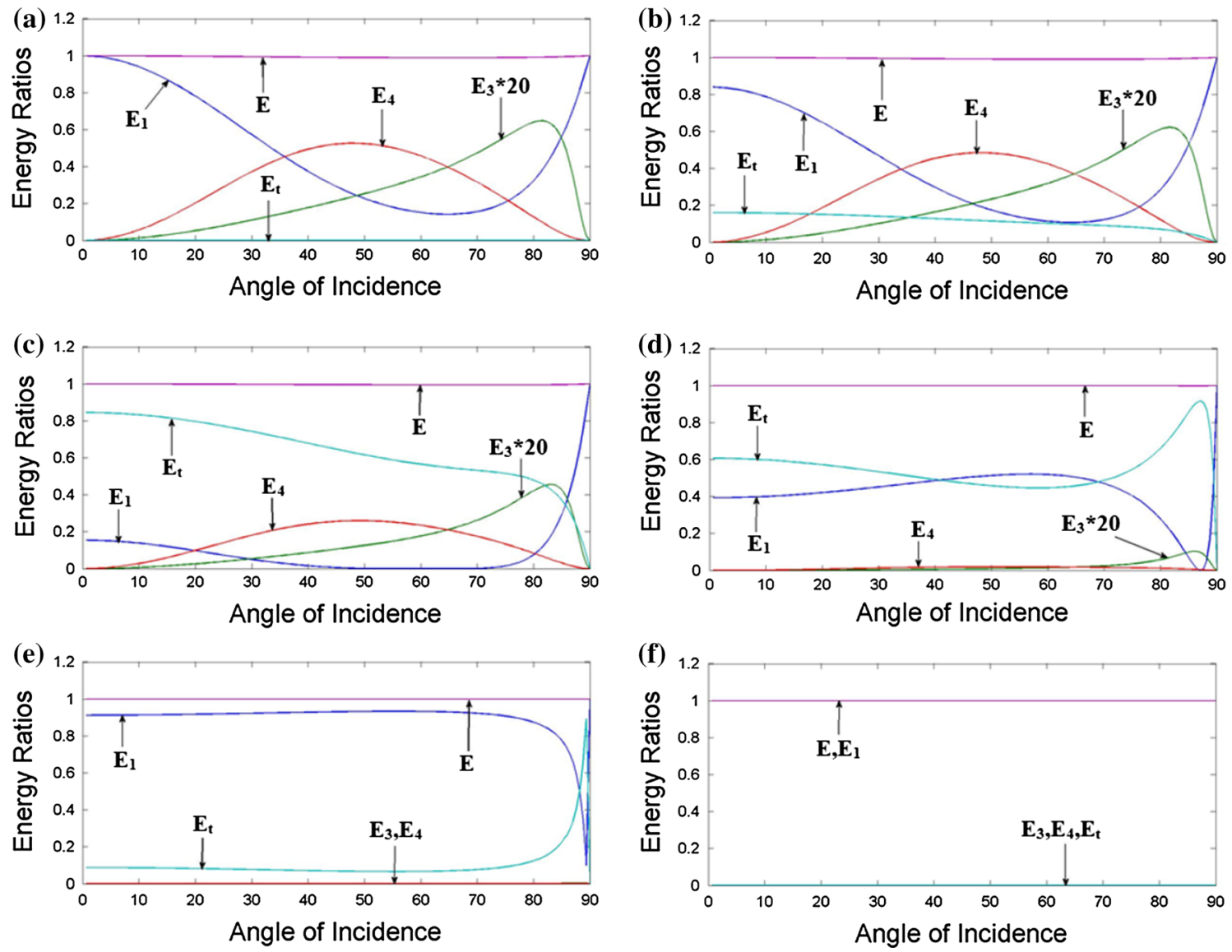

Fig. 3 Influences of normal stiffness $S_{1}$ on the reflection coefficients. a $S_{1}=10^{5}$; b $S_{1}=10^{11}$; c $S_{1}=10^{12}$; d $S_{1}=10^{13}$; e $S_{1}=10^{14} ; \mathbf{f} S_{1}=10^{20}$

The averaged energy flux in one period is defined as

$$
\bar{P}_{i}=\frac{\omega}{2 \pi} \int_{-\pi / \omega}^{\pi / \omega} P_{i}(t) \mathrm{d} t=\frac{1}{2} \operatorname{Re}\left(\dot{u}_{j}^{*} \sigma_{j i}+\dot{\varphi}_{j}^{*} m_{i j}\right),
$$

where the superscript “ $*$ ” in $\dot{u}_{i}^{*}$ and $\dot{\varphi}_{i}^{*}$ indicates the conjugated complex. The energy flux along the propagation direction $\mathbf{n}$ is

$$
\bar{P}_{n}(\mathbf{n})=\bar{P}_{x} \cos (\mathbf{n}, x)+\bar{P}_{y} \cos (\mathbf{n}, y) .
$$

Define the reflection coefficients as the energy flux ratios of the reflection waves to the incident wave.

$$
E_{s}=\frac{\bar{P}_{n}^{R}\left(\mathbf{n}_{s}\right)}{\bar{P}_{n}^{I}\left(\mathbf{n}_{0}\right)} ; \quad(s=1,3,4),
$$

where $\bar{P}_{n}^{I}\left(\mathbf{n}_{0}\right)$ is the energy flux of the incident wave, and $\bar{P}_{n}^{R}\left(\mathbf{n}_{s}\right)(s=1,3,4)$ is the energy flux of the reflection LD wave, CTDR3 wave and CTDR4 wave.

Considering that a thin layer at surface with the length of one wave length $2 l(l=\pi / k)$ and the infinitesimal thickness $\delta \rightarrow 0$. The energy conservation within the thin layer requires that the input energy flux is equal to the output energy flux, namely

$$
E=\left(\bar{P}_{z}^{R}\left(\mathbf{n}_{1}\right)+\bar{P}_{z}^{R}\left(\mathbf{n}_{3}\right)+\bar{P}_{z}^{R}\left(\mathbf{n}_{4}\right)+\bar{P}_{z}^{t}\right) / \bar{P}_{z}^{I}\left(\mathbf{n}_{0}\right)=1
$$

where $\bar{P}_{z}^{I}\left(\mathbf{n}_{0}\right)$ is the input energy flux carried by incident wave. $\bar{P}_{z}^{R}\left(\mathbf{n}_{1}\right), \bar{P}_{z}^{R}\left(\mathbf{n}_{3}\right)$, and $\bar{P}_{z}^{R}\left(\mathbf{n}_{4}\right)$ are the output energy fluxes carried by reflection LD wave, CTDR3 wave, and CTDR4 waves, respectively. $\bar{P}_{z}^{t}=$ 

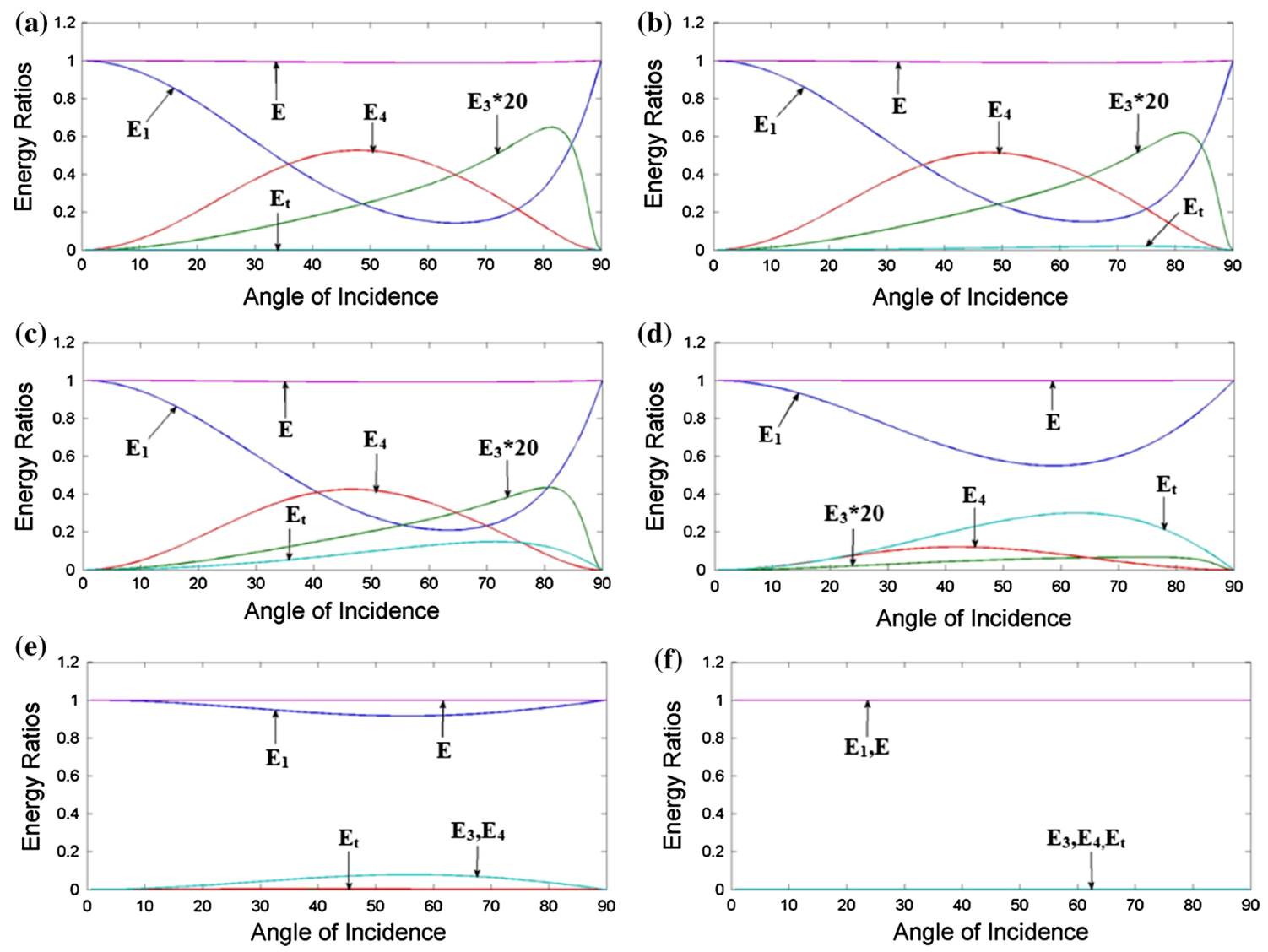

Fig. 4 Influences of tangent stiffness $S_{2}$ on the reflection coefficients. a $S_{2}=10^{5}$; b $S_{2}=10^{10}$; c $S_{2}=10^{11}$; d $S_{2}=10^{12}$; e $S_{2}=10^{13} ; \mathbf{f} S_{2}=10^{20}$

$\frac{1}{2}\left(\dot{u}_{z} S_{1} u_{z}+\dot{u}_{y} S_{2} u_{y}+\dot{\varphi}_{x} S_{3} \varphi_{x}\right)$ and $E_{t}=\bar{P}_{z}^{t} / \bar{P}_{z}^{I}$ stand for the energy flux and energy flux ratio stored in the constraint springs. Equation (20) can be used to validate the numerical results in the next section.

\section{Numerical results and discussions}

In the numerical examples, the material constants of micropolar elastic half-space are $\rho=2600 \mathrm{~kg} / \mathrm{m}^{3}, \lambda=$ $2.2 \times 10^{10} \mathrm{~Pa}, \mu=1.1 \times 10^{10} \mathrm{~Pa}, K=8.8 \times 10^{8} \mathrm{~Pa}, \gamma=2 \times 10^{8} \mathrm{~N}, j=6 \times 10^{-7} \mathrm{~m}^{2}$, which are taken from [10]. Because the coupled transverse displacement and transverse microrotational waves (CTDR3 and CTDR4) are both dispersive, the reflection CTDR3 wave and the reflection CTDR4 wave are not only dependent on the incident angle but also on the circular frequency of the incident wave. Considering that the influence of the circular frequency on the reflection amplitude ratio was already discussed in [6], the incident circular frequency is assumed fixed in the present work, namely $\omega^{2} / \omega_{0}^{2}=8$, which is above the cutoff frequency of CTDR3 wave. The reflection coefficients in terms of energy flux ratio are calculated in the present work for different incident angles in the case of incident LD wave and incident CTDR3 wave. In order to investigate the influences of constraint on the reflection energy partition, some finite values of constraint stiffness coefficients between 0 and $\infty$ are used.

\subsection{In the case of incident LD wave}

Figure 3 shows the influence of the normal stiffness $S_{1}$ on the reflection energy flux partition. The reflection coefficients are calculated for normal stiffness $S_{1}=10^{5}, 10^{11}, 10^{12}, 10^{13}, 10^{14}$, and $10^{20}$, respectively, while keeping the tangent stiffness and the rotational stiffness zero, namely $S_{2}=S_{3}=0$. It is observed that the reflection coefficients of CTDR3 wave and the CTDR4 wave decrease gradually as the normal stiffness $S_{1}$ 

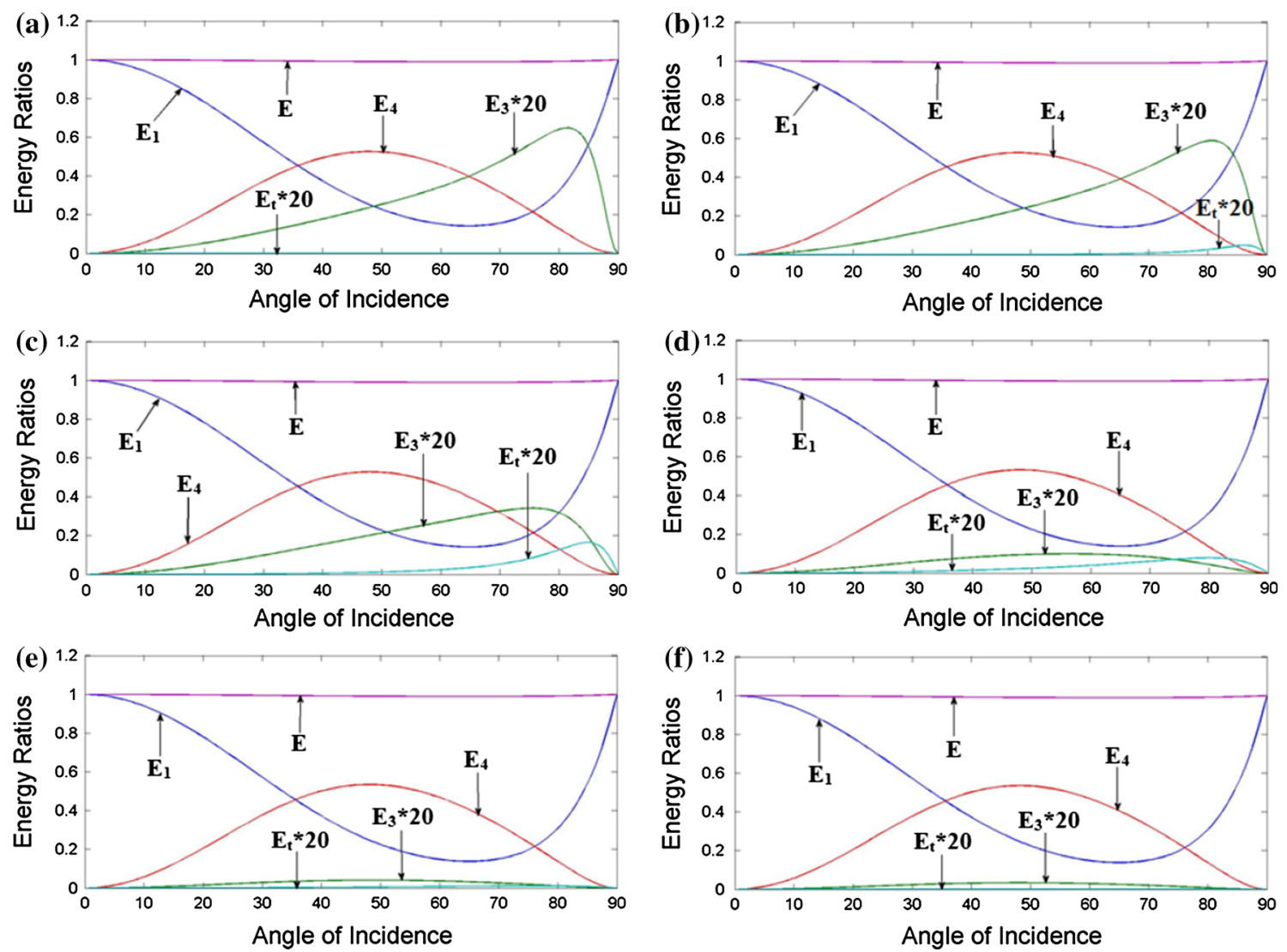

Fig. 5 Influences of rotational stiffness $S_{3}$ on the reflection coefficients. a $S_{3}=10^{5} ; \mathbf{b} S_{3}=10^{8} ; \mathbf{c} S_{3}=10^{9} ; \mathbf{d} S_{3}=10^{10}$; e $S_{3}=10^{11} ; \mathbf{f} S_{3}=10^{20}$
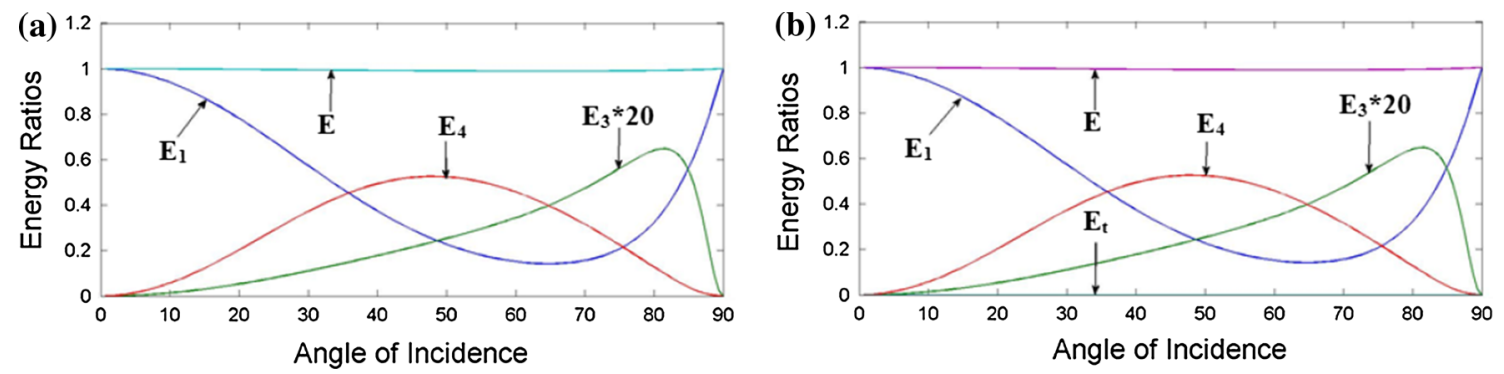

Fig. 6 Reflection coefficients for the free surface and the constraint surface with very small constraint stiffness $\left(S_{1}=S_{2}=S_{3}=\right.$ $\left.10^{0}\right)$. a The free surface, $\mathbf{b}$ the constraint surface

increases. However, the reflection coefficient of LD wave decreases first and then increases gradually as the normal stiffness increases. The reflection coefficients of $L D$ wave reach minimum around $S_{1}=10^{12}$. Although the influences of the normal stiffness $S_{1}$ on LD wave are opposite before and after $S_{1}=10^{12}$, the increase in $S_{1}$ always suppress the energy flux of CTDR3 wave and CTDR4 wave. This means that the existence of normal stiffness suppresses the mode conversion.

Figure 4 shows the influence of the tangent stiffness coefficients on the reflection energy flux partition. The reflection coefficients are calculated for tangent stiffness $S_{2}=10^{5}, 10^{10}, 10^{11}, 10^{12}, 10^{13}$, and $10^{20}$, respectively, while keeping the normal stiffness and the rotational stiffness zero, namely $S_{1}=S_{3}=0$. It is found that the tangent stiffness $S_{2}$ can influence all kinds of reflection waves. The reflection coefficient of LD wave increases gradually, and the reflection coefficients of the CTDR3 wave and the CTDR4 wave decrease gradually as the tangent stiffness $S_{2}$ increases. This means that the tangent constraint can also suppress the mode conversion as the normal constraint. Moreover, the reflection coefficients are most sensitive to the 

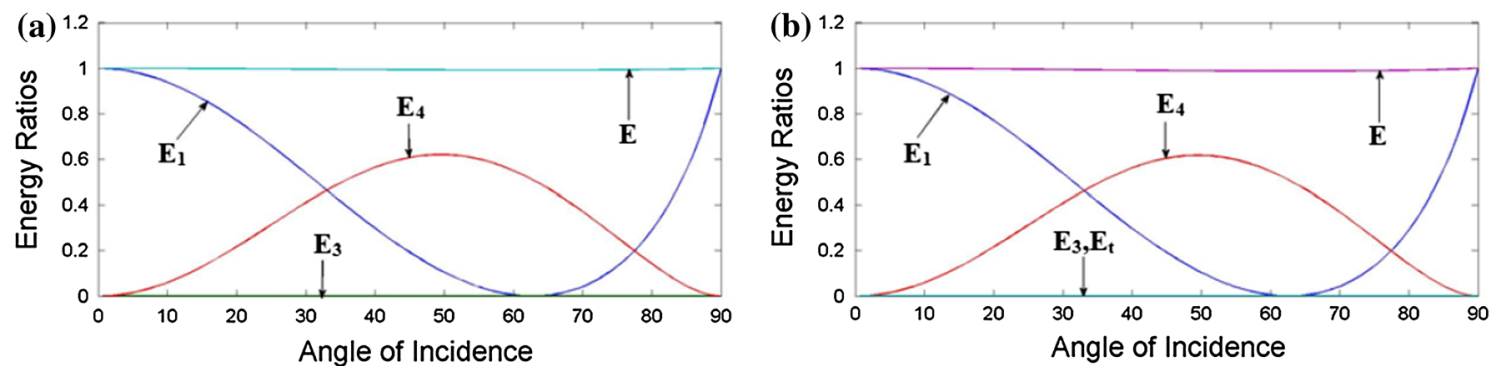

Fig. 7 Reflection coefficients for the fixed surface and the constraint surface with very large constraint stiffness $\left(S_{1}=S_{2}=S_{3}=\right.$ $\left.10^{20}\right)$. a The fixed surface, $\mathbf{b}$ the constraint surface
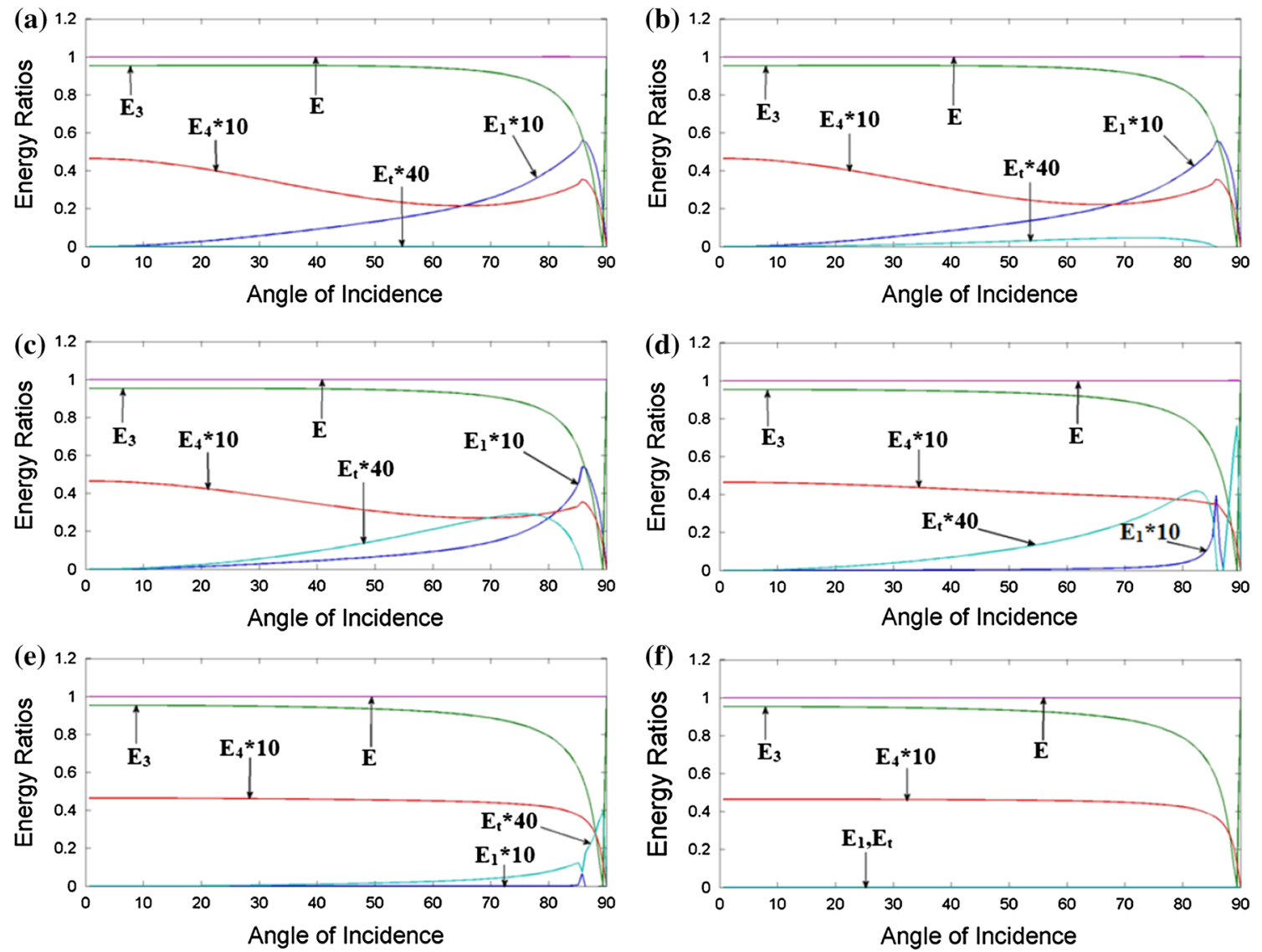

Fig. 8 Influences of normal stiffness $S_{1}$ on the reflection coefficients. a $S_{1}=10^{5}$; b $S_{1}=10^{11}$; c $S_{1}=10^{12}$; d $S_{1}=10^{13}$; e $S_{1}=10^{14} ; \mathbf{f} S_{1}=10^{20}$

tangent stiffness around $S_{2}=10^{11}-10^{12}$. When the tangent stiffness coefficient is very small or very large, the reflection coefficients are insensitive to stiffness coefficient.

Figure 5 shows the influence of rotational stiffness on the reflection energy partition. The reflection coefficients are calculated for rotational stiffness $S_{3}=10^{5}, 10^{8}, 10^{9}, 10^{10}, 10^{11}$, and $10^{20}$, respectively, while keeping the normal stiffness and the tangent stiffness fixed, namely $S_{1}=S_{2}=0$. Different from the normal and tangent stiffness, the rotational stiffness has only evident influence on the CTDR3 wave, while it has nearly unnoticed influences on the LD wave and the CTDR4 wave. This means that the existence of rotational stiffness $S_{3}$ only suppresses the CTDR3 wave.

In order to validate the numerical results about reflection energy flux ratios, the energy flux conservation is checked up. The energy flux conservation principle requires the output energy fluxes are equal to the input energy flux at same area of non-free surface. The curves indicated by " $E$ " in Figs. 3, 4, and 5 keep nearly unit in total range of incident angle, which implies that the energy flux conservation is satisfied in total range of 

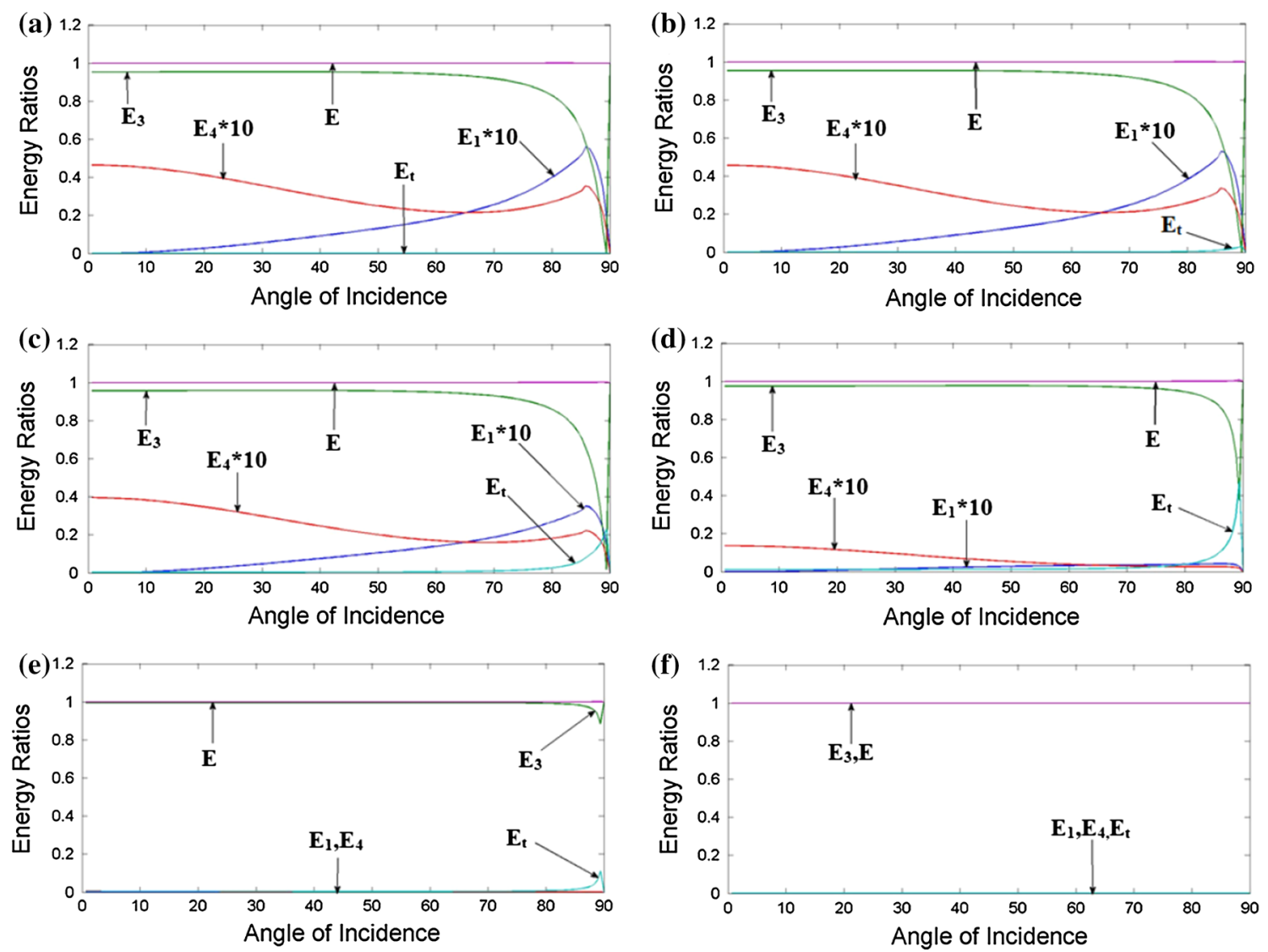

Fig. 9 Influences of tangent stiffness $S_{2}$ on the reflection coefficients. a $S_{2}=10^{5}$; b $S_{2}=10^{10}$; c $S_{2}=10^{11}$; d $S_{2}=10^{12}$; e $S_{2}=10^{13} ; \mathbf{f} S_{2}=10^{20}$

incident angles. The curves indicated by " $E_{\mathrm{t}}$ " in Figs. 3, 4, and 5 represent the energy stored in the constraint spring, which is evidently dependent on the constraint stiffness. By observing Figs. 3, 4, and 5, it is noted that the normal constrain spring stores energy most evidently around $S_{1}=10^{12} \sim 10^{13}$, the tangent constraint spring stores energy most evidently around $S_{2}=10^{11} \sim 10^{12}$, and the rotational constraint spring stores energy most evidently around $S_{3}=10^{9} \sim 10^{10}$. By comparison, the normal constraint spring stores energy most evidently, while the rotational constraint spring stores energy least evidently.

The free surface was often used to study the reflection problem in the previous literatures. It is actually a limiting case of the present non-free surface model when the normal, tangent, and rotational constraint stiffness, $S_{1}, S_{2}$, and $S_{3}$ all tend to zero. Therefore, the reflection coefficients for the free surface can be recovered from the present non-free surface model by assigning very small values to $S_{1}, S_{2}$, and $S_{3}$. Figure 6 shows the reflection coefficients obtained from the free surface accurately and obtained from present model by assigning very small value to $S_{1}, S_{2}$, and $S_{3}$. It is found that there is a good consistence between them. Similarly, the fixed surface can be recovered also as another limiting case of present non-free surface model when the normal, tangent, and rotational constraint stiffness, $S_{1}, S_{2}$, and $S_{3}$ all tend to infinity. Figure 7 shows the reflection coefficients obtained from the fixed surface accurately and obtained from the present non-free surface by assigning very large values to $S_{1}, S_{2}$, and $S_{3}$. It is also found that there is a good consistence between them. This can also be a validity of present numerical results.

\subsection{In the case of incident CTDR3 wave}

Figure 8 shows the dependence of reflection coefficients on the normal stiffness $S_{1}$ in the case of incident CTDR3 wave. It is observed that the reflection coefficient of LD wave decreases gradually, and the reflection coefficient of the CTDR4 wave increases gradually with increasing normal stiffness $S_{1}$. The reflection coefficient of the 

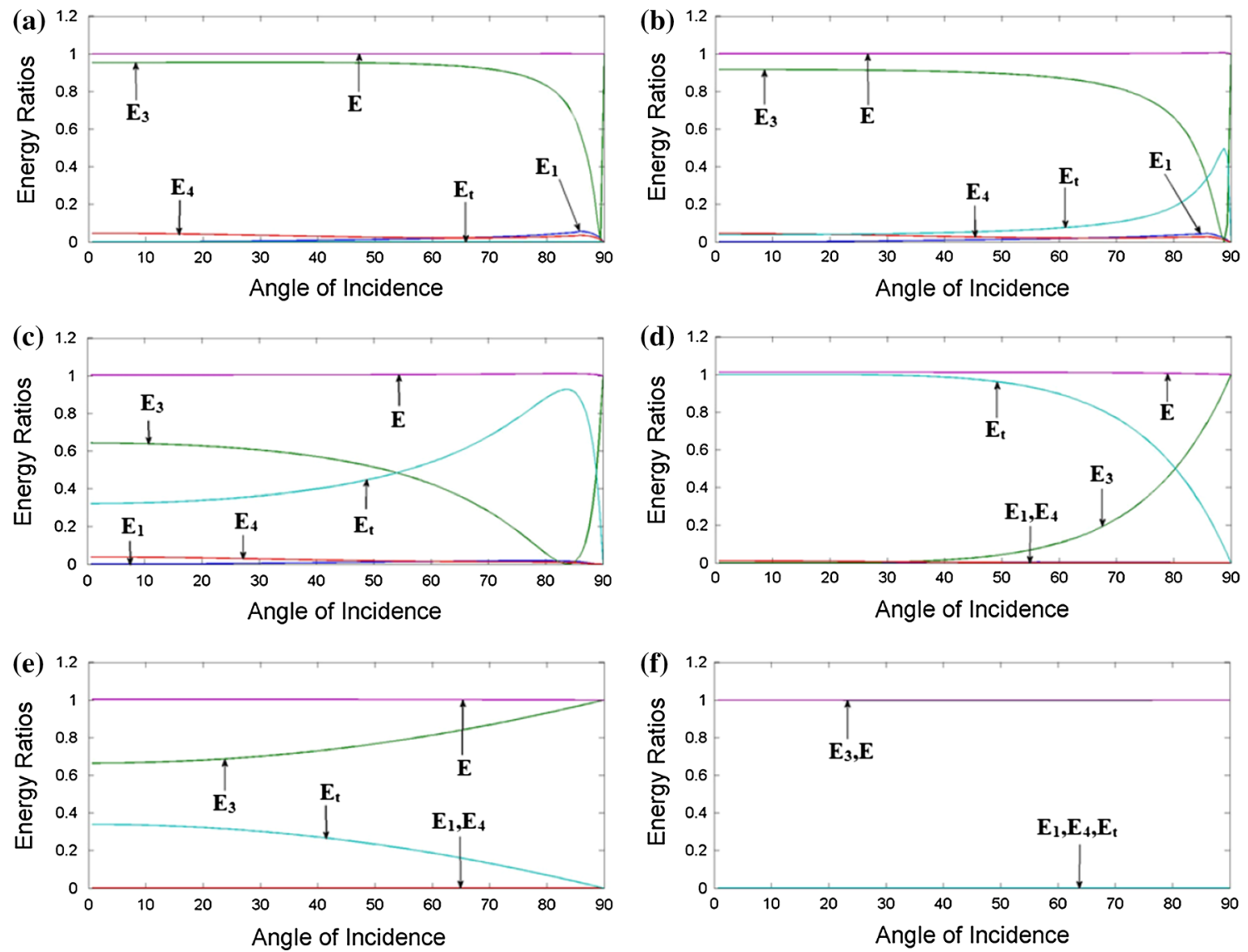

Fig. 10 Influences of rotational stiffness $S_{3}$ on the reflection coefficients. a $S_{3}=10^{5} ; \mathbf{b} S_{3}=10^{8} ; \mathbf{c} S_{3}=10^{9} ; \mathbf{d} S_{3}=10^{10}$; e $S_{3}=10^{11} ; \mathbf{f} S_{3}=10^{20}$

CTDR3 wave keeps nearly independent of the normal stiffness. This observation implies that the larger normal stiffness does not help the mode conversion.

Figure 9 shows the dependence of reflection coefficients on the tangent stiffness $S_{2}$. As in the case of incident LD wave, the tangent stiffness influences all types of reflection waves. It is observed that the reflection coefficients of LD wave and CTDR4 wave decrease, while the reflection coefficient of the CTDR3 wave increases gradually with increasing tangent stiffness $S_{2}$. Compared with the normal stiffness $S_{1}$, the tangent stiffness has more evident influences on the CTDR4 wave and thus has more evident suppressant effect on the mode conversion.

Figure 10 shows the dependence of reflection coefficients on the rotational stiffness $S_{3}$. As in the case of incident LD wave, the rotational stiffness has only evident influence on the CTDR3 wave, while it has nearly unnoticed influences on the LD wave and CTDR4 wave. When the rotational stiffness $S_{3}$ increases, the reflection coefficient of the CTDR3 wave decreases first and then increases gradually after about $S_{3}=10^{10}$. Moreover, it is observed that the reflection coefficient of the CTDR4 wave becomes very small when the rotational stiffness $S_{3}$ is not zero. This means that the existence of rotational stiffness has evident suppressant effect on the reflection CTDR4 wave.

In order to validate the energy flux conservation, incident energy flux and the reflection energy flux along the normal surface and the energy flux stored in the constraint spring are also calculated. The curves indicated by " $E$ " in Figs. 8, 9, and 10 keep nearly unit in total range of incident angle, which implies that the energy flux conservation is satisfied in total range of incident angles. However, different from the case of incident LD wave, the rotational constraint stiffness stores energy most evidently from the observation on curves indicated by " $E_{\mathrm{t}}$ " in Figs. 8, 9, and 10. This phenomenon can be explained by that the dilatational deformation dominates in the deformation response to the incident LD wave and thus the normal constraint spring stores energy most 
evidently, while the microrotational deformation dominates in the deformation response to the incident CTDR3 wave and thus the rotational constraint spring stores energy most evidently.

\section{Conclusions}

The non-free surface of a micropolar elastic half-space is a generalized surface model. The free surface, slippery surface, and the fixed surface can be recovered by the non-free surface model. At the non-free surface, the surface macrotraction and microcouple are assumed to be proportional to the surface macrodisplacement and surface microrotation. The normal, tangent, and rotational stiffness represent the mechanical behavior of non-free surface and have different influences on the reflection waves. (a) The rotational stiffness has only evident influence on the CTDR3 wave; however, the normal and tangent stiffness have influences on all types of reflection waves. (b) The tangent stiffness has evident suppressant effects on the CTDR4 wave, but has opposite influences on the LD wave and the CTDR3 wave for both incident LD wave and incident CTDR3 wave. (c) The normal stiffness suppresses the CTDR4 wave for incident LD wave but enhances CTDR4 wave for incident CTDR3 wave. The mode conversion phenomena are most evident at the free surface and disappear at the fixed surface. The non-free surface has evident suppressant effect on the mode conversion compared with the free surface. The normal and tangent constraints suppress all types of reflection waves, while the rotational constraint only suppresses the reflection CTDR3 wave. Moreover, different from the free surface and the fixed surface, the non-free surface can store energy and therefore makes the normal energy flux of all reflection waves not equal to that of incident waves. The amplitude of energy stored in the constrained spring is dependent on the specific value of stiffness coefficients, which can be deliberately designed to adjust the energy fluxes carried by various reflection waves. Due to the deformation responses to incident LD wave and incident CTDR3 wave are different, the normal constraint, the tangent constraint, and the rotational constraint play different roles in storing energy.

Acknowledgments The work is supported by the National Natural Science Foundation of China (No. 10972029) and Opening fund of State Key Laboratory of Nonlinear Mechanics (LNM)

\section{Appendix A}

In the case of non-free surface, the explicit expressions of each element in matrix $\mathbf{D}$ in Eq. (13) are

$$
\begin{aligned}
& d_{11}=-\left[\lambda+(2 \mu+K) \cos ^{2} \theta_{1}^{R}\right]\left(k_{1}^{R}\right)^{2}-\cos \theta_{1}^{R} k_{1}^{R} S_{1}, \\
& d_{12}=(2 \mu+K) \sin \theta_{3}^{R} \cos \theta_{3}^{R}\left(k_{3}^{R}\right)^{2}+\sin \theta_{3}^{R} k_{3}^{R} S_{1}, \\
& d_{13}=(2 \mu+K) \sin \theta_{4}^{R} \cos \theta_{4}^{R}\left(k_{4}^{R}\right)^{2}+\sin \theta_{4}^{R} k_{4}^{R} S_{1}, \\
& d_{21}=(2 \mu+K) \sin \theta_{1}^{R} \cos \theta_{1}^{R}\left(k_{1}^{R}\right)^{2}+\sin \theta_{1}^{R} k_{1}^{R} S_{2}, \\
& d_{22}=\left[\left(\mu \cos 2 \theta_{3}^{R}+K \cos ^{2} \theta_{3}^{R}\right)\left(k_{3}^{R}\right)^{2}+\frac{K \omega_{0}^{2}}{V_{3}^{2}-2 \omega_{0}^{2} /\left(k_{3}^{R}\right)^{2}-c_{4}^{2}}\right]+\cos \theta_{3}^{R} k_{3}^{R} S_{2} \text {, } \\
& d_{23}=\left[\left(\mu \cos 2 \theta_{4}^{R}+K \cos ^{2} \theta_{4}^{R}\right)\left(k_{4}^{R}\right)^{2}+\frac{K \omega_{0}^{2}}{V_{4}^{2}-2 \omega_{0}^{2} /\left(k_{4}^{R}\right)^{2}-c_{4}^{2}}\right]+\cos \theta_{4}^{R} k_{4}^{R} S_{2} \text {, } \\
& d_{31}=0, \quad d_{32}=-\frac{\gamma \omega_{0}^{2} \cos \theta_{3}^{R} k_{3}^{R}}{V_{3}^{2}-2 \omega_{0}^{2} /\left(k_{3}^{R}\right)^{2}-c_{4}^{2}}+\frac{\omega_{0}^{2} S_{3}}{V_{3}^{2}-2 \omega_{0}^{2} /\left(k_{3}^{R}\right)^{2}-c_{4}^{2}}, \\
& d_{33}=-\frac{\gamma \omega_{0}^{2} \cos \theta_{4}^{R} k_{4}^{R}}{V_{4}^{2}-2 \omega_{0}^{2} /\left(k_{4}^{R}\right)^{2}-c_{4}^{2}}+\frac{\omega_{0}^{2} S_{3}}{V_{4}^{2}-2 \omega_{0}^{2} /\left(k_{4}^{R}\right)^{2}-c_{4}^{2}} .
\end{aligned}
$$

In the case of free surface, the explicit expressions of each element in matrix $\mathbf{D}$ are

$$
d_{11}=-\left[\lambda+(2 \mu+K) \cos ^{2} \theta_{1}^{R}\right]\left(k_{1}^{R}\right)^{2}, \quad d_{12}=(2 \mu+K) \sin \theta_{3}^{R} \cos \theta_{3}^{R}\left(k_{3}^{R}\right)^{2},
$$




$$
\begin{aligned}
& d_{13}=(2 \mu+K) \sin \theta_{4}^{R} \cos \theta_{4}^{R}\left(k_{4}^{R}\right)^{2}, \quad d_{21}=(2 \mu+K) \sin \theta_{1}^{R} \cos \theta_{1}^{R}\left(k_{1}^{R}\right)^{2}, \\
& d_{22}=\left[\left(\mu \cos 2 \theta_{3}^{R}+K \cos ^{2} \theta_{3}^{R}\right)\left(k_{3}^{R}\right)^{2}+\frac{K \omega_{0}^{2}}{V_{3}^{2}-2 \omega_{0}^{2} /\left(k_{3}^{R}\right)^{2}-c_{4}^{2}}\right], \\
& d_{23}=\left[\left(\mu \cos 2 \theta_{4}^{R}+K \cos ^{2} \theta_{4}^{R}\right)\left(k_{4}^{R}\right)^{2}+\frac{K \omega_{0}^{2}}{V_{4}^{2}-2 \omega_{0}^{2} /\left(k_{4}^{R}\right)^{2}-c_{4}^{2}}\right], \\
& d_{31}=0, d_{32}=-\frac{\gamma \omega_{0}^{2} \cos \theta_{3}^{R} k_{3}^{R}}{V_{3}^{2}-2 \omega_{0}^{2} /\left(k_{3}^{R}\right)^{2}-c_{4}^{2}}, \quad d_{33}=-\frac{\gamma \omega_{0}^{2} \cos \theta_{4}^{R} k_{4}^{R}}{V_{4}^{2}-2 \omega_{0}^{2} /\left(k_{4}^{R}\right)^{2}-c_{4}^{2}} .
\end{aligned}
$$

$\mathbf{E}_{\text {free }}$ can be obtained from Eqs. (15a) and (15b) by letting $S_{1}, S_{2}$, and $S_{3}$ equal to zero, respectively. In the case of fixed surface, the explicit expressions of each element in matrix $\mathbf{D}$ are

$$
\begin{aligned}
& d_{11}=\cos \theta_{1}^{R} k_{1}^{R}, \quad d_{12}=-\sin \theta_{3}^{R} k_{3}^{R}, \quad d_{13}=-\sin \theta_{4}^{R} k_{4}^{R}, \quad d_{21}=\sin \theta_{1}^{R} k_{1}^{R}, \quad d_{22}=\cos \theta_{3}^{R} k_{3}^{R}, \\
& d_{23}=\cos \theta_{4}^{R} k_{4}^{R}, \quad d_{31}=0, \quad d_{32}=\frac{\omega_{0}^{2}}{V_{3}^{2}-2 \omega_{0}^{2} /\left(k_{3}^{R}\right)^{2}-c_{4}^{2}}, \quad d_{33}=\frac{\omega_{0}^{2}}{V_{4}^{2}-2 \omega_{0}^{2} /\left(k_{4}^{R}\right)^{2}-c_{4}^{2}},
\end{aligned}
$$

$\mathbf{E}_{\text {fixed }}=\left(\cos \theta_{1}^{I} k_{1}^{I},-\sin \theta_{1}^{I} k_{1}^{I}, 0\right)^{T}$ for incident LD wave, and

$\mathbf{E}_{\text {fixed }}=\left(\sin \theta_{p}^{I} k_{p}^{I}, \cos \theta_{p}^{I} k_{p}^{I},-\frac{\omega_{0}^{2}}{V_{p}^{2}-2 \omega_{0}^{2} /\left(k_{p}^{I}\right)^{2}-c_{4}^{2}}\right)^{T}$ for incident CTDR3 wave $(p=3)$ or CTDR4 wave $(p=4)$.

\section{References}

1. Ariman, T.: Wave propagation in a micropolar elastic half-space. Acta Mech. 13(1-2), 11-20 (1972)

2. Chiriţă, S., Ghiba, I.D.: Rayleigh waves in Cosserat elastic materials. Int. J. Eng. Sci. 51, 117-127 (2012)

3. Eringen, A.C.: Linear theory of micropolar elasticity. Indiana Univ. Math. J. 15(6), 909-923 (1966)

4. Khurana, A., Tomar, S.K.: Propagation of plane elastic waves at a plane interface between two electro-microelastic solid half-spaces. Int. J. Solids Struct. 44(11), 3773-3795 (2007)

5. Kumar, R., Sharma, N., Ram, P.: Interfacial imperfection on reflection and transmission of plane waves in anisotropic micropolar media. Theor. Appl. Fract. Mech. 49(3), 305-312 (2008)

6. Parfitt, V.R., Eringen, A.C.: Reflection of plane waves from the flat boundary of a micropolar elastic half-space. J. Acoust. Soc. Am. 45(5), 1258-1272 (1969)

7. Singh, B.: Wave propagation in an orthotropic micropolar elastic solid. Int. J. Solids Struct. 44(11), 3638-3645 (2007)

8. Smith, A.C.: Waves in micropolar elastic solids. Int. J. Eng. Sci. 5(10), 741-746 (1967)

9. Tomar, S.K., Gogna, M.L.: Reflection and refraction of a longitudinal microrotational wave at an interface between two micropolar elastic solids in welded contact. Int. J. Eng. Sci. 30(11), 1637-1646 (1992)

10. Tomar, S.K., Gogna, M.L., Reflection and refraction of longitudinal wave at an interface between two micropolar elastic solids at welded contact. J. Acoust. Soc. Am. 97(2), 822-830 (1995). [Erratum: in J. Acoust. Soc. Am. 102, 2452 (1997)]

11. Tomar, S.K., Gogna, M.L.: Reflection and refraction of coupled transverse and micro-rotational waves at an interface between two different micropolar elastic welded contact. Int. J. Eng. Sci. 33(4), 485-496 (1995)

12. Tomar, S.K., Kumar, R.: Wave propagation at liquid/micropolar elastic solid interface. J. Sound Vib. 222(5), 858-869 (1999)

13. Tomar, S.K., Khurana, A.: Elastic waves in an electro-microelastic solid. Int. J. Solids Struct. 45(1), 276-302 (2008) 